\title{
Extinction in Nonautonomous Discrete Lotka-Volterra Competitive System with Pure Delays and Feedback Controls
}

\author{
Ling Zhang, ${ }^{1}$ Zhidong Teng, ${ }^{1}$ Tailei Zhang, ${ }^{1}$ and Shujing Gao ${ }^{2}$ \\ ${ }^{1}$ College of Mathematics and System Sciences, Xinjiang University, Urumqi 830046, China \\ ${ }^{2}$ College of Mathematics and Computer Science, Gannan Normal University, Ganzhou 341000, China \\ Correspondence should be addressed to Zhidong Teng, zhidong1960@163.com
}

Received 25 June 2009; Accepted 12 October 2009

Recommended by Elena Braverman

The paper discusses a nonautonomous discrete time Lotka-Volterra competitive system with pure delays and feedback controls. New sufficient conditions for which a part of the $n$-species is driven to extinction are established by using the method of multiple discrete Lyapunov functionals.

Copyright (C) 2009 Ling Zhang et al. This is an open access article distributed under the Creative Commons Attribution License, which permits unrestricted use, distribution, and reproduction in any medium, provided the original work is properly cited.

\section{Introduction}

The coexistence and global stability of population models are of the interesting subjects in mathematical biology. Many authors have argued that the discrete time models are governed by differential equations which are more appropriate than the continuous ones to describe the dynamics of population when the population has nonoverlapping generations, a lot has been done on discrete Lotka-Volterra systems.

May in [1] firstly considered the following autonomous discrete two-species LotkaVolterra competitive system:

$$
\begin{aligned}
& x(n+1)=x(n) \exp \left\{r_{1}-a_{11} x(n)-a_{12} y(n)\right\}, \\
& y(n+1)=y(n) \exp \left\{r_{2}-a_{21} x(n)-a_{22} y(n)\right\}
\end{aligned}
$$

and studied the stable points, stable cycles, and the chaos behaivor. Further, Lu and Wang [2] studied the permanence and global attractivity of this system. 
Chen and Zhou in [3] considered the following periodic discrete two-species LotkaVolterra competitive system:

$$
\begin{gathered}
x(n+1)=x(n) \exp \left\{r_{1}\left(1-\frac{x(n)}{K_{1}(n)}-\mu_{2}(n) y(n)\right)\right\}, \\
y(n+1)=y(n) \exp \left\{r_{2}\left(1-\mu_{1} x(n)-\frac{y(n)}{K_{2}(n)}\right)\right\}
\end{gathered}
$$

and studied the permanence and existence of a periodic solution, and further, sufficient conditions are established on the global stability of the periodic solution.

Zhang and Zhou in [4] investigated the following nonautonomous discrete twospecies Lotka-Volterra competitive system:

$$
\begin{gathered}
x(n+1)=x(n) \exp \left\{r_{1}(n)-a_{11}(n) x\left(n-k_{1}\right)-a_{12}(n) y\left(n-k_{2}\right)\right\}, \\
y(n+1)=y(n) \exp \left\{r_{2}(n)-a_{21}(n) x\left(n-l_{1}\right)-a_{22}(n) y\left(n-l_{2}\right)\right\} .
\end{gathered}
$$

Some sufficient conditions were obtained for the permanence of the system.

Wang et al. in [5] studied the following general nonautonomous discrete $n$-species Lotka-Volterra systems:

$$
x_{i}(k+1)=x_{i}(k) \exp \left\{r_{i}(k)-\sum_{j=1}^{n} \sum_{l=0}^{m} a_{i j}^{l}(k) x_{j}(k-l)-a_{12}(n) y\left(n-k_{2}\right)\right\}, \quad i=1,2, \ldots, n
$$

By applying the linear method and constructing the appropriate Lyapunov functionals, the author established the sufficient conditions which guarantee that any positive solution of this system is stable and attracts others, and obtained some applications of main results.

Muroya in [6,7] considered the following general nonautonomous discrete $n$-species Lotka-Volterra systems:

$$
\begin{gathered}
N_{i}(p+1)=N_{i}(p) \exp \left\{c_{i}(p)-a_{i}(p) N_{i}(p)-\sum_{j=1}^{n} \sum_{l=0}^{m} a_{i j}^{l}(p) N_{j}\left(p-k_{l}\right)\right\}, \quad p=0,1,2, \ldots, \\
N_{i}(s)=N_{i s} \geq 0 \quad \forall s \leq 0, N_{i 0}>0, \quad i=1,2, \ldots, n,
\end{gathered}
$$

and related pure delays models, that is, $a_{i}(p)=0$ for all $p \geq 0$. The author obtained the permanence and the global asymptotically stable by applying mean-value conditions and the method of constructing discrete Lyapunov functionals. 
Liao et al. in [8] discussed the following general discrete nonautonomous $n$-species competitive system with feedback controls:

$$
\begin{gathered}
x_{i}(k+1)=x_{i}(k) \exp \left\{b_{i}(k)-\sum_{j=1}^{n} a_{i j}(k) x_{j}(k)-\sum_{j=1, j \neq i}^{n} c_{i j}(k) x_{i}(k) x_{j}(k)-d_{i}(k) u_{i}(k)\right\}, \quad k=0,1,2, \ldots, \\
\Delta u_{i}(k)=r_{i}(k)-e_{i}(k) u_{i}(k)+f_{i}(k) x_{i}(k), \quad i=1,2, \ldots, n .
\end{gathered}
$$

Some sufficient conditions are established on the permanence and the global stability of the system.

Recently, we see that in $[9,10]$ the authors studied the following nonautonomous continuous Lotka-Volterra competitive system with pure delays and feedback controls:

$$
\begin{gathered}
\dot{x}(t)=x_{i}(t)\left[r_{i}(t)-\sum_{j=1}^{n} a_{i j}(t) x_{j}\left(t-\tau_{i j}(t)\right)-\sum_{j=1}^{n} \int_{-\sigma_{i j}}^{0} b_{i j}(t, s) x_{j}(t+s) d s\right. \\
\left.\quad-c_{i}(t) u_{i}(t)-d_{i}(t) u_{i}\left(t-\tau_{i}(t)\right)\right], \\
\dot{u}(t)=-e_{i}(t) u_{i}(t)+f_{i}(t) x_{i}(t)+g_{i}(t) x_{i}\left(t-\delta_{i}(t)\right), \quad i=1,2, \ldots, n .
\end{gathered}
$$

The sufficient conditions for which a part of the $n$-species is driven to extinction and the surplus part of the $n$-species remains permanence are established.

However, we see that for general discrete $n$-species population systems the results for which a part of the $n$-species is driven to extinction and the surplus part of the $n$-species remains the permanence, up to now, are still not obtained. Therefore, motivated by the above works, in this paper we study the following discrete nonautonomous Lotka-Volterra competitive system with pure delays and feedback controls

$$
\begin{aligned}
& x_{i}(k+1)=x_{i}(k) \exp \left\{r_{i}(k)-\sum_{j=1}^{n} a_{i j}(k) x_{j}\left(k-\tau_{i j}\right)\right. \\
& \left.-\sum_{j=1}^{n} \sum_{l=0}^{\sigma_{i j}} b_{i j l}(k) x_{j}(k-l)-d_{i}(k) u_{i}\left(k-\tau_{i}\right)\right\}, \\
& u_{i}(k+1)=\left(1-e_{i}(k)\right) u_{i}(k)+g_{i}(k) x_{i}\left(k-\delta_{i}\right), \quad i=1,2, \ldots, n .
\end{aligned}
$$

The main purpose is to establish a criterion for which guarantee the part species $x_{r+1}, x_{r+2}, \ldots, x_{n}$ in system (1.8) is driven to extinction. The method used in this paper is to constructing the multiple discrete Lyapunov functions. On the permanence of the surplus species $x_{1}, x_{2}, \ldots, x_{r}$, owing to the length of this paper, we will give the discussion in another paper. 
This paper is organized as follows. In next section, as preliminaries, some assumptions and useful lemmas are introduced. In Section 3, the main results of this paper on the extinction of a part of the $n$-species of system (1.8) are established. In Section 4 , an example is presented to illustrate the feasibility of our results.

\section{Preliminaries}

Let $Z$ denote the set of all nonnegative integers. For any bounded sequence $y(k)$, we denote $\bar{y}=\sup _{k \in Z}\{y(k)\}, \underline{y}=\inf _{k \in Z}\{y(k)\}$. Throughout this paper, we introduce the following assumptions.

$\left(H_{1}\right) r_{i}(k)$ is a bounded sequence defined on $Z ; a_{i j}(k), d_{i}(k), g_{i}(k)$ and $b_{i j l}(k)(i, j=$ $\left.1,2, \ldots, n, l=0,1, \ldots, \sigma_{i j}\right)$ are nonnegative bounded sequences defined on $Z ; \tau_{i j}$, $\tau_{i}, \delta_{i}$, and $\sigma_{i j}(i, j=1,2, \ldots, n)$ are nonnegative integers.

$\left(H_{2}\right)$ Sequences $e_{i}(k)(i=1,2, \ldots, n)$ satisfy $0<e_{i}(k) \leq 1$ for all $k \in Z$.

$\left(H_{3}\right)$ There exist positive integers $\omega$ and $\lambda$ such that for each $i=1,2, \ldots, n$

$$
\liminf _{n \rightarrow \infty} \sum_{s=n}^{n+\omega-1} r_{i}(s)>0, \quad \liminf _{n \rightarrow \infty} \sum_{s=n}^{n+\lambda-1}\left[a_{i i}(s)+\sum_{l=0}^{\sigma_{i i}} b_{i i l}(s)\right]>0 .
$$

$\left(H_{4}\right)$ There exists positive integer $\beta$ such that for each $i=1,2, \ldots, n$

$$
\limsup _{n \rightarrow \infty} \prod_{n}^{n+\beta-1}\left(1-e_{i}(s)\right)<1 .
$$

Let $R_{+}=[0, \infty)$ and $R_{+}^{n}=\left\{x=\left(x_{1}, x_{2}, \ldots, x_{n}\right): x_{i} \in R_{+}, i=1,2, \ldots, n\right\}$. We denote by int $R_{+}^{n}$ the interior of $R_{+}^{n}$. For any nonnegative constants $a$ and $b$ with $a<b$, we denote by $[a, b]_{Z}$ the set of all integers in the interval $[a, b]$. For some integer $m \geq 0$, we denote by $D_{+}[-m, 0]_{Z}$ the space of all nonnegative discrete time function $\phi:[-m, 0]_{Z} \rightarrow R_{+}^{n}$ with norm $\|\phi\|=\sup \left\{|\phi(s)|: s \in[-m, 0]_{Z}\right\}$.

Let $\tau=\max \left\{\tau_{i j}, \tau_{i}, \delta_{i}, \sigma_{i j}: i, j=1,2, \ldots, n\right\}$. Motivated by the biological background of system (1.8), in this paper we only consider all solutions of system (1.8) that satisfy the following initial conditions:

$$
x_{i}(s)=\phi_{i}(s) \geq 0, \quad u_{i}(s)=\psi_{i}(s) \geq 0, \quad s \in[-m, 0]_{Z}, i=1,2, \ldots, n,
$$

where $\phi=\left(\phi_{1}, \phi_{2}, \ldots, \phi_{n}\right) \in D_{+}[-m, 0]_{Z}$ and $\psi=\left(\psi_{1}, \psi_{2}, \ldots, \psi_{n}\right) \in D_{+}[-m, 0]_{Z}$. For any $\phi, \psi \in D_{+}[-m, 0]_{Z}$, let $z=(\phi, \psi)$, by the fundamental theory of difference equations, system (1.8) has a unique solution $(x(s, z),(s, z))$ satisfying the initial condition $(2.3)$, where $x(s, z)=$ $\left(x_{1}(s, z), x_{2}(s, z), \ldots, x_{n}(s, z)\right)$ and $u(s, z)=\left(u_{1}(s, z), u_{2}(s, z), \ldots, u_{n}(s, z)\right)$. It is obvious that solution $(x(s, z),(s, z))$ is positive, that is, $x_{i}(s, z)>0$ and $u_{i}(s, z)>0(i=1,2, \ldots, n)$ for all $s \in Z$.

We first consider the following nonautonomous difference inequality system:

$$
y(n+1) \leq y(n) \exp \{\alpha(n)-\beta(n) y(n)\}, \quad n \in Z,
$$


where $\alpha(n)$ and $\beta(n)$ are bounded sequences defined on $Z$ and $\beta(n) \geq 0$ for all $n \in Z$. We have the following result.

Lemma 2.1 (see [11]). Assume that there exists an integer $\lambda>0$ such that

$$
\liminf _{n \rightarrow \infty} \sum_{k=n}^{n+\lambda} \beta(k)>0,
$$

then there exists a constant $M>0$ such that for any nonnegative solution $y(n)$ of system (2.4) with initial value $y\left(n_{0}\right)=y_{0} \geq 0$, where $n_{0} \in Z$ is some integer,

$$
\limsup _{n \rightarrow \infty} y(n)<M
$$

Next, we consider the following nonautonomous linear difference equation:

$$
v(n+1)=\gamma(n) v(n)+\omega(n),
$$

where $\gamma(n)$ and $\omega(n)$ are nonnegative bounded sequences defined on $Z$. We have the following results.

Lemma 2.2 (see [11]). Assume that there exists an integer $\lambda>0$ such that

$$
\limsup _{n \rightarrow \infty} \prod_{k=n}^{n+\lambda} r(k)<1
$$

then there exists a constant $M>0$ such that for any nonnegative solution $v(n)$ of system (2.7) with initial value $v\left(n_{0}\right)=v_{0} \geq 0$, where $n_{0} \in Z$ is some integer,

$$
\limsup _{n \rightarrow \infty} v(n)<M
$$

Lemma 2.3 (see [11]). Assume that the conditions of Lemma 2.2 hold, then for any constants $\varepsilon>0$ and $M_{1}>0$ there exist positive constants $\widehat{\delta}=\widehat{\delta}(\varepsilon)$ and $\widehat{n}=\widehat{n}\left(\varepsilon, M_{1}\right)$ such that for any $\widehat{n}_{0} \in Z$ and $0 \leq v_{0} \leq M_{1}$, when $\omega(n)<\widehat{\delta}$ for all $n \geq \widehat{n}_{0}$, one has

$$
v\left(n, \widehat{n}_{0}, v_{0}\right)<\varepsilon \quad \forall n \geq \widehat{n}_{0}+\widehat{n},
$$

where $v\left(n, \widehat{n}_{0}, v_{0}\right)$ is the solution of (2.7) with initial value $v\left(\widehat{n}_{0}\right)=v_{0}$.

Lemma 2.4. Assume that assumptions $\left(H_{1}\right)-\left(H_{4}\right)$ hold, then there exists a constant $M_{0}>0$ such that

$$
\limsup _{k \rightarrow \infty} x_{i}(k)<M_{0}, \quad \limsup _{k \rightarrow \infty} u_{i}(k)<M_{0}, \quad i=1,2, \ldots, n
$$

for any positive solution $\left(x_{1}(k), x_{2}(k), \ldots, x_{n}(k), u_{1}(k), u_{2}(k), \ldots, u_{n}(k)\right)$ of system (1.8). 
Proof. Let $\left(x_{1}(k), x_{2}(k), \ldots, x_{n}(k), u_{1}(k), u_{2}(k), \ldots, u_{n}(k)\right)$ be any positive solution of system (1.8). For each $i \in\{1,2, \ldots, n\}$, we have

$$
\begin{aligned}
x_{i}(k+1) & =x_{i}(k) \exp \left\{r_{i}(k)-\sum_{j=1}^{n} a_{i j}(k) x_{j}\left(k-\tau_{i j}\right)-\sum_{j=1}^{n} \sum_{l=0}^{\sigma_{i j}} b_{i j l}(k) x_{j}(k-l)-d_{i}(k) u_{i}\left(k-\tau_{i}\right)\right\} \\
& \leq x_{i}(k) \exp \left\{r_{i}(k)\right\},
\end{aligned}
$$

then, for any integer $k \geq 0$ and $\theta \leq 0$ with $k+\theta \geq 0$, summing inequality (2.12) from $k+\theta$ to $k-1$, we obtain

$$
x_{i}(k+\theta) \geq x_{i}(k) \exp \left\{-\sum_{s=k+\theta}^{k-1} r_{i}(s)\right\}
$$

Therefore, for any integer $k \geq \tau$, from (2.13) and the first equation of system (1.8) we obtain

$$
\begin{gathered}
x_{i}(k+1) \leq x_{i}(k) \exp \left\{r_{i}(k)-a_{i i}(k) x_{i}\left(k-\tau_{i i}\right)-\sum_{l=0}^{\sigma_{i i}} b_{i i l}(k) x_{i}(k-l)\right\} \\
\leq x_{i}(k) \exp \left\{r_{i}(k)-a_{i i}(k) x_{i}(k) \exp \left\{-\sum_{s=k+\theta}^{k-1} r_{i}(s)\right\}\right. \\
\left.-\sum_{l=0}^{\sigma_{i i}} b_{i i l}(k) x_{i}(k) \exp \left\{-\sum_{s=k-l}^{k-1} r_{i}(s)\right\}\right\} .
\end{gathered}
$$

Since for any $k \geq \tau$ and $l \in[0, \tau]_{Z}$

$$
\sum_{s=k+\theta}^{k-1} r_{i}(s) \leq \tau r_{i}^{u}
$$

where $r_{i}^{u}=\sup _{k \in Z} r_{i}(k)$, we have from (2.14),

$$
x_{i}(k+i) \leq x_{i}(k) \exp \left\{r_{i}(k)-h_{i}\left(a_{i i}(k)+\sum_{l=0}^{\sigma_{i i}} b_{i i l}(k)\right) x_{i}(k)\right\}
$$

for any $k \geq \tau$, where $h_{i}=\exp \left\{-\tau r_{i}^{u}\right\}$.

We consider the following auxiliary equation:

$$
y_{i}(k+1) \leq y_{i}(k) \exp \left\{r_{i}(k)-\beta_{i}(k) y_{i}(k)\right\}, \quad k \in Z,
$$


where $\beta_{i}(k)=h_{i}\left(a_{i i}(k)+\sum_{l=0}^{\sigma_{i i}} b_{i i l}(k)\right)$, then by assumption $\left(H_{3}\right)$ and applying Lemma 2.1 there exists a constant $N_{i}>0$ such that

$$
\limsup _{k \rightarrow \infty} x_{i}(k)<N_{i}
$$

for any positive solution $y_{i}(k)$ of (2.17). Therefore, from the comparison theorem of difference equation, we finally obtain

$$
\limsup _{k \rightarrow \infty} x_{i}(k)<N_{i}
$$

Further form inequality (2.19), there exists a positive constant $k_{i} \geq \tau$ such that

$$
x_{i}(k)<N_{i}, \quad x_{i}\left(k-\delta_{i}\right)<N_{i} \quad \forall k \geq k_{i}
$$

Thus, from the second equation of system (1.8), we obtain

$$
u_{i}(k+1) \leq\left(1-e_{i}(k)\right) u_{i}(k)+\left(f_{i}(k)+g_{i}(k)\right) N_{i}
$$

for all $k \geq k_{i}$. We consider the following auxiliary equation:

$$
v_{i}(k+1)=\left(1-e_{i}(k)\right) v_{i}(k)+\left(f_{i}(k)+g_{i}(k)\right) N_{i}
$$

then by assumption $\left(H_{4}\right)$ and applying Lemma 2.2, there exists a constant $N_{i}^{*}$ such that

$$
\limsup _{k \rightarrow \infty} v_{i}(k)<N_{i}^{*}
$$

for any positive solution $v_{i}(k)$ of (2.22). Let $v_{i}^{*}(k)$ be the solution of (2.22) with initial value $v_{i}^{*}\left(k_{i}\right)=u_{i}\left(k_{i}\right)$, then from the comparison theorem of difference equation, we have $u_{i}(k) \leq$ $v_{i}^{*}\left(k_{i}\right)$ for all $k \geq k_{i}$. Thus, we finally obtain

$$
\limsup _{k \rightarrow \infty} u_{i}(k)<N_{i}^{*}
$$

Let $N=\max _{1 \leq i \leq n}\left\{N_{i}, N_{i}^{*}\right\}$, then from (2.19) and (2.24) we finally see that the conclusions of Lemma 2.4 hold. 


\section{Main Results}

In this section, we discuss the extinction of the part of species $x_{r+1}, \ldots, x_{n}$ of system (1.8).

Define functions as follows:

$$
\begin{gathered}
A_{i j}(k)=a_{i j}\left(k+\tau_{i j}\right)+\sum_{l=0}^{\sigma_{i j}} b_{i j l}(k+l), \\
D_{i}(k)=d_{i}\left(k+\tau_{i}\right), \quad R_{i}(k)=g_{i}\left(k+\delta_{i}\right),
\end{gathered}
$$

where $i, j=1,2, \ldots, n$.

Theorem 3.1. Assume that assumptions $\left(H_{1}\right)-\left(H_{4}\right)$ hold and there exists an integer $1 \leq r<n$ such that for any $h>r$ there exists an integer $i_{h}<h$ such that

$$
\begin{gathered}
\limsup _{k \rightarrow \infty} \frac{\sum_{s=k}^{k+\omega-1} r_{h}(s)}{\sum_{s=k}^{k+\omega-1} r_{i_{h}}(s)}<\liminf _{k \rightarrow \infty} \frac{A_{h j}(k)}{A_{i_{h} j}(k)} \quad \forall j \leq h, \\
\liminf _{k \rightarrow \infty} \frac{D_{h}(k)}{e_{h}(k)}>\limsup _{k \rightarrow \infty}\left(\frac{A_{i_{h} h}(k)}{R_{h}(k)} \limsup _{k \rightarrow \infty} \frac{\sum_{s=k}^{k+\omega-1} r_{h}(s)}{\sum_{s=k}^{k+\omega-1} r_{i_{h}}(s)}-\frac{A_{h h}(k)}{R_{h}(k)}\right), \\
\limsup _{k \rightarrow \infty} \frac{D_{i_{h}}(k)}{e_{i_{h}}(k)}<\liminf _{k \rightarrow \infty}\left(\frac{A_{h_{h}}(k)}{R_{i_{h}}(k)} \liminf _{k \rightarrow \infty} \frac{\sum_{s=k}^{k+\omega-1} r_{i_{h}}(s)}{\sum_{s=k}^{k+\omega-1} r_{h}(s)}-\frac{A_{i_{h} i_{h}}(k)}{R_{i_{h}}(k)}\right),
\end{gathered}
$$

then for each $i=r+1, \ldots, n$ one has

$$
\lim _{k \rightarrow \infty} x_{i}(k)=0, \quad \lim _{k \rightarrow \infty} u_{i}(k)=0, \quad \sum_{k=0}^{\infty} x_{i}(k)<\infty
$$

for any positive solution $\left(x_{1}(k), x_{2}(k), \ldots, x_{n}(k), u_{1}(k), u_{2}(k), \ldots, u_{n}(k)\right)$ of system (1.8).

Proof. From assumption $\left(H_{2}\right)$, there exist constant $\eta_{0}>0$ and integer $K_{0}>0$ such that

$$
\sum_{s=k}^{k+\omega-1} r_{i}(s) \geq \eta_{0} \quad \forall k \geq K_{0}
$$


We first prove that $\lim _{k \rightarrow \infty} x_{n}(k)=0$. Let $h=n$ and $i_{h}=p$. From conditions (3.2) we can find positive constants $\alpha_{n 1}, \alpha_{n 2}, \alpha_{n 3}, \alpha_{n 4}, \varepsilon_{n}$ and integer $K_{n} \geq K_{0}$ such that

$$
\begin{aligned}
& \frac{\sum_{s=k}^{k+\omega-1} r_{n}(s)}{\sum_{s=k}^{k+\omega-1} r_{p}(s)}<\frac{\alpha_{n 1}}{\alpha_{n 2}}-\varepsilon_{n}<\frac{\alpha_{n 1}}{\alpha_{n 2}}<\frac{A_{n j}(k)}{A_{p j}(k)}, \\
& \frac{D_{n}(k)}{e_{n}(k)}>\frac{\alpha_{n 4}}{\alpha_{n 2}}>\frac{\alpha_{n 1} A_{p n}(k)-\alpha_{n 2} A_{n n}(k)}{\alpha_{n 2} R_{n}(k)}, \\
& \frac{D_{p}(k)}{e_{p}(k)}<\frac{\alpha_{n 3}}{\alpha_{n 1}}<\frac{\alpha_{n 2} A_{n p}(k)-\alpha_{n 1} A_{p p}(k)}{\alpha_{n 1} R_{p}(k)}
\end{aligned}
$$

for all $k \geq K_{n}$ and $j=1,2, \ldots, n$. Consequently,

$$
\begin{gathered}
\sum_{s=k}^{k+\omega-1}\left(-\alpha_{n 1} r_{p}(s)+\alpha_{n 2} r_{n}(s)\right)<-\varepsilon_{n} \alpha_{n 2} \eta_{0} \\
\alpha_{n 1} A_{p j}(k)-\alpha_{n 2} A_{n j}(k)<0 \\
\alpha_{n 4} e_{n}(k)-\alpha_{n 2} D_{n}(k)<0 \\
\alpha_{n 1} A_{p p}(k)-\alpha_{n 2} A_{n p}(k)+\alpha_{n 3} R_{p}(k)<0 \\
-\alpha_{n 3} e_{p}(k)+\alpha_{n 1} D_{p}(k)<0 \\
\alpha_{n 1} A_{p n}(k)-\alpha_{n 2} A_{n n}(k)-\alpha_{n 4} R_{n}(k)<0
\end{gathered}
$$

for all $k \geq K_{n}$ and $j=1,2, \ldots, n$.

Let $(x(k), u(k))=\left(x_{1}(k), x_{2}(k), \ldots, x_{n}(k), u_{1}(k), u_{2}(k), \ldots, u_{n}(k)\right)$ be any positive solution of system (1.8). Constructing the following discrete Lyapunov functional

$$
\begin{aligned}
& V_{n}(k)=x_{p}^{-\alpha_{n 1}}(k) x_{n}^{\alpha_{n 2}}(k) \\
& \quad \times \exp \left\{\alpha_{n 3} u_{p}(k)-\alpha_{n 4} u_{n}(k)\right. \\
& \quad+\alpha_{n 1} \sum_{j=1}^{n} \sum_{s=k-\tau_{p j}}^{k-1} a_{p j}\left(s+\tau_{p j}\right) x_{j}(s)+\alpha_{n 1} \sum_{j=1}^{n} \sum_{l=0}^{\sigma_{p j}} \sum_{s=k-l}^{k-1} b_{p j l}(s+l) x_{j}(s) \\
& \quad+\alpha_{n 1} \sum_{s=k-\tau_{p}}^{k-1} d_{p}\left(s+\tau_{p}\right) u_{p}(s)-\alpha_{n 2} \sum_{j=1}^{n} \sum_{s=k-\tau_{n j}}^{k-1} a_{n j}\left(s+\tau_{n j}\right) x_{j}(s) \\
& \quad-\alpha_{n 2} \sum_{j=1}^{n} \sum_{l=0}^{\sigma_{n j}} \sum_{s=k-l}^{k-1} b_{n j l}(s+l) x_{j}(s)-\alpha_{n 2} \sum_{s=k-\tau_{n}}^{k-1} d_{n}\left(s+\tau_{n}\right) u_{n}(s) \\
& \left.\quad+\alpha_{n 3} \sum_{s=k-\delta_{p}}^{k-1} g_{p}\left(s+\delta_{p}\right) x_{p}(s)-\alpha_{n 4} \sum_{s=k-\delta_{n}}^{k-1} g_{n}\left(s+\delta_{n}\right) x_{n}(s)\right\}
\end{aligned}
$$


By calculating, we obtain

$$
\begin{aligned}
& \frac{V_{n}(k+1)}{V_{n}(k)} \\
& =\exp \left\{-\alpha_{n 1}\left[r_{p}(k)-\sum_{j=1}^{n} a_{p j}(k) x_{j}\left(k-\tau_{p j}\right)-\sum_{j=1}^{n} \sum_{l=0}^{\sigma_{p j}} b_{p j l}(k) x_{j}(k+l)-d_{p}(k) u_{p}\left(k-\tau_{p}\right)\right]\right. \\
& +\alpha_{n 2}\left[r_{n}(k)-\sum_{j=1}^{n} a_{n j}(k) x_{j}\left(k-\tau_{n j}\right)-\sum_{j=1}^{n} \sum_{l=0}^{\sigma_{n j}} b_{n j l}(k) x_{j}(k+l)-d_{n}(k) u_{n}\left(k-\tau_{n}\right)\right] \\
& +\alpha_{n 3}\left[-e_{p}(k) u_{p}(k)+g_{p}(k) x_{p}\left(k-\delta_{p}\right)\right]-\alpha_{n 4}\left[-e_{n}(k) u_{n}(k)+g_{n}(k) x_{n}\left(k-\delta_{n}\right)\right] \\
& +\alpha_{n 1} \sum_{j=1}^{n}\left[a_{p j}\left(k+\tau_{p j}\right) x_{j}(k)-a_{p j}(k) x_{j}\left(k-\tau_{p j}\right)\right] \\
& +\alpha_{n 1} \sum_{j=1}^{n} \sum_{l=0}^{\sigma_{p j}}\left[b_{p j l}(k+l) x_{j}(k)-b_{p j l}(k) x_{j}(k-l)\right] \\
& +\alpha_{n 1}\left[d_{p}\left(k+\tau_{p}\right) u_{p}(k)-d_{p}(k) u_{p}\left(k-\tau_{p}\right)\right] \\
& -\alpha_{n 2} \sum_{j=1}^{n}\left[a_{n j}\left(k+\tau_{n j}\right) x_{j}(k)-a_{n j}(k) x_{j}\left(k-\tau_{n j}\right)\right] \\
& -\alpha_{n 2} \sum_{j=1}^{n} \sum_{l=0}^{\sigma_{n j}}\left[b_{n j l}(k+l) x_{j}(k)-b_{n j l}(k) x_{j}(k-l)\right] \\
& -\alpha_{n 2}\left[d_{n}\left(k+\tau_{n}\right) u_{n}(k)-d_{n}(k) u_{n}\left(k-\tau_{n}\right)\right] \\
& +\alpha_{n 3}\left[g_{p}\left(k+\delta_{p}\right) x_{p}(k)-g_{p}(k) x_{p}\left(k-\delta_{p}\right)\right] \\
& \left.-\alpha_{n 4}\left[g_{n}\left(k+\delta_{n}\right) x_{n}(k)-g_{n}(k) x_{n}\left(k-\delta_{n}\right)\right]\right\} \\
& =\exp \left\{-\alpha_{n 1} r_{p}(k)+\alpha_{n 2} r_{n}(k)-\left(\alpha_{n 3} e_{p}(k)-\alpha_{n 1} D_{p}(k)\right) u_{p}(k)\right. \\
& -\left(\alpha_{n 2} D_{n}(k)-\alpha_{n 4} e_{n}(k)\right) u_{n}(k)+\sum_{j \neq p}^{n-1}\left(\alpha_{n 1} A_{p j}(k)-\alpha_{n 2} A_{n j}(k)\right) x_{j}(k) \\
& +\left(\alpha_{n 1} A_{p p}(k)-\alpha_{n 2} A_{n p}(k)-\alpha_{n 3} R_{p}(k)\right) x_{p}(k) \\
& \left.+\left(\alpha_{n 1} A_{p n}(k)-\alpha_{n 2} A_{n n}(k)-\alpha_{n 4} R_{n}(k)\right) x_{n}(k)\right\} \text {. }
\end{aligned}
$$


From inequalities (3.7)-(3.11), we can obtain

$$
V_{n}(k+1) \leq V_{n}(k) \exp \left\{-\alpha_{n 1} r_{p}(k)+\alpha_{n 2} r_{n}(k)\right\} \quad \forall k \geq K_{n}
$$

For any $k>K_{n}$, we choose an integer $m \geq 0$, such that $k \in\left[K_{n}+m \omega, K_{n}+(m+1) \omega\right)$, then from (3.6) and (3.14) we further have

$$
\begin{aligned}
V_{n}(k) & \leq V_{n}\left(K_{n}\right) \exp \left\{\sum_{s=K_{n}}^{k-1}\left(-\alpha_{n 1} r_{p}(s)+\alpha_{n 2} r_{n}(s)\right)\right\} \\
& \leq V_{n}\left(K_{n}\right) \exp \left\{\left(\sum_{s=K_{n}}^{K_{n}+m \omega-1}+\sum_{s=K_{n}+m \omega}^{k-1}\right)\left(-\alpha_{n 1} r_{p}(s)+\alpha_{n 2} r_{n}(s)\right)\right\} \\
& \leq V_{n}\left(K_{n}\right) \exp \left\{-\varepsilon_{n} \alpha_{n 2} \eta_{0} m+M_{n}\right\} \\
& \leq V_{n}\left(K_{n}\right) \exp \left\{-\lambda_{n} k+M_{n}^{*}\right\},
\end{aligned}
$$

where

$$
\begin{gathered}
\lambda_{n}=\frac{\varepsilon_{n} \alpha_{n 2} \eta_{0}}{\omega}, \quad M_{n}^{*}=M_{n}+\varepsilon_{n} \alpha_{n 2} \eta_{0}\left(1+\frac{K_{n}}{\omega}\right), \\
M_{n}=\sup _{k \in Z}\left(-\alpha_{n 1} r_{p}(k)+\alpha_{n 2} r_{n}(k)\right) \omega .
\end{gathered}
$$

On the other hand, from assumptions $\left(H_{1}\right)$, we have

$$
\begin{aligned}
V_{n}(k) \geq & x_{p}^{-\alpha_{n 1}}(k) x_{n}^{\alpha_{n 2}}(k) \\
& \times \exp \left\{-\alpha_{n 4} u_{n}(k)-\sum_{j=1}^{n} \alpha_{n 2} \sum_{s=k-\tau_{n j}}^{k-1} a_{n j}\left(s+\tau_{n j}\right) x_{j}(s)\right. \\
& -\sum_{j=1}^{n} \alpha_{n 2} \sum_{l=0}^{\sigma_{n j}} \sum_{s=k-l}^{k-1} b_{n j l}(s+l) x_{j}(s)-\alpha_{n 2} \sum_{s=k-\tau_{n}}^{k-1} d_{n}\left(s+\tau_{n}\right) u_{n}(s) \\
& \left.-\alpha_{n 4} \sum_{s=k-\delta_{n}}^{k-1} g_{n}\left(s+\delta_{n}\right) x_{n}(s)\right\} \\
\geq & x_{p}^{-\alpha_{n 1}}(k) x_{n}^{\alpha_{n 2}}(k) \\
\times & \quad \exp \left\{-\alpha_{n 4} M_{0}-\alpha_{n 2} \tau \sum_{j=1}^{n} \overline{a_{n j}} M_{0}\right. \\
& \left.-\alpha_{n 2} \sum_{j=1}^{n} \sum_{l=0}^{\sigma_{n j}} \overline{b_{n j l}} l M_{0}-\alpha_{n 2} \tau \overline{d_{n}} M_{0}-\alpha_{n 4} \tau \overline{g_{n}} M_{0}\right\}
\end{aligned}
$$


for all $k \geq \tau$, where constant $M_{0}$ is given in Lemma 2.4. Hence, there exist a positive constant $Q_{n}>0$ such that

$$
V_{n}(k) \geq x_{p}^{-\alpha_{n 1}}(k) x_{n}^{\alpha_{n 2}}(k) Q_{n} \quad \forall k \geq \tau .
$$

From (3.15) and (3.18), we obtain

$$
\begin{aligned}
x_{n}(k) & \leq\left[Q_{n}^{-1} x_{p}^{\alpha_{n 1}}(k) V_{n}\left(K_{n}\right) \exp \left\{-\lambda_{n} k+M_{n}^{*}\right\}\right]^{1 / \alpha_{n 2}} \\
& \leq Q_{n}^{*} \exp \left\{-\lambda_{n}^{*} k\right\}
\end{aligned}
$$

for all $k \geq K_{n}+\tau$, where

$$
Q_{n}^{*}=\sup _{k \in Z}\left[x_{p}^{\alpha_{n 1}}(k) V_{n}\left(K_{n}\right) Q_{n}^{-1} \exp M_{n}^{*}\right]^{1 / \alpha_{n 2}}, \quad \lambda_{n}^{*}=\frac{\lambda_{n}}{\alpha_{n 2}} .
$$

From (3.19), we finally obtain

$$
\lim _{k \rightarrow \infty} x_{n}(k)=0, \quad \sum_{k=0}^{\infty} x_{n}(k)<\infty
$$

Next, we consider the second equation of system (1.8), applying Lemma 2.3 we can easily obtain $u_{n}(k) \rightarrow 0$ as $k \rightarrow \infty$.

Now, we suppose that for any $t>r$, we have obtained

$$
\lim _{k \rightarrow \infty} x_{i}(k)=0, \quad \lim _{k \rightarrow \infty} u_{i}(k)=0, \quad \sum_{k=0}^{\infty} x_{n}(k)<\infty
$$

for all $i>t$. We further will prove

$$
\lim _{k \rightarrow \infty} x_{t}(k)=0, \quad \sum_{k=0}^{\infty} x_{t}(k)<\infty
$$

From conditions (3.2), we can choose positive constants $\alpha_{t 1}, \alpha_{t 2}, \alpha_{t 3}, \alpha_{t 4}, \varepsilon_{t}$ and integer $K_{t} \geq K_{n}$ such that

$$
\begin{aligned}
& \frac{\sum_{s=k}^{k+\omega-1} r_{t}(s)}{\sum_{s=k}^{k+\omega-1} r_{q}(s)}<\frac{\alpha_{t 1}}{\alpha_{t 2}}-\varepsilon_{t}<\frac{\alpha_{t 1}}{\alpha_{t 2}}<\frac{A_{t j}(k)}{A_{q j}(k)} \\
& \frac{D_{t}(k)}{e_{t}(k)}>\frac{\alpha_{t 4}}{\alpha_{t 2}}>\frac{\alpha_{t 1} A_{q n}(k)-\alpha_{t 2} A_{t t}(k)}{\alpha_{t 2} R_{t}(k)} \\
& \frac{D_{q}(k)}{e_{q}(k)}<\frac{\alpha_{t 3}}{\alpha_{t 1}}>\frac{\alpha_{t 2} A_{t q}(k)-\alpha_{t 1} A_{q q}(k)}{\alpha_{t 1} R_{q}(k)}
\end{aligned}
$$


for all $k \geq K_{t}$ and $j=1,2, \ldots, t$, where $i_{h}=q$. Consequently,

$$
\begin{gathered}
\sum_{s=k}^{k+\omega-1}\left(-\alpha_{t 1} r_{q}(s)+\alpha_{t 2} r_{t}(s)\right)<-\varepsilon_{t} \alpha_{t 2} \eta_{0}, \\
\alpha_{t 1} A_{q j}(k)-\alpha_{t 2} A_{t j}(k)<0, \\
\alpha_{t 4} e_{t}(k)-\alpha_{t 2} D_{t}(k)<0, \\
\alpha_{t 1} A_{q q}(k)-\alpha_{t 2} A_{t q}(k)+\alpha_{t 3} R_{q}(k)<0, \\
-\alpha_{t 3} e_{q}(k)+\alpha_{t 1} D_{q}(k)<0 \\
\alpha_{t 1} A_{q t}(k)-\alpha_{t 2} A_{t t}(k)-\alpha_{t 4} R_{t}(k)<0
\end{gathered}
$$

for all $k \geq K_{t}$ and $j=1,2, \ldots, t$. Constructing the following discrete Lyapunov functional

$$
\begin{aligned}
& V_{t}(k)=x_{q}^{-\alpha_{t 1}}(k) x_{t}^{\alpha_{t 2}}(k) \\
& \times \exp \left\{\alpha_{t 3} u_{q}(k)-\alpha_{t 4} u_{t}(k)\right. \\
& \\
& \quad+\alpha_{t 1} \sum_{j=1}^{n} \sum_{s=k-\tau_{q j}}^{k-1} a_{q j}\left(k+\tau_{q j}\right) x_{j}(s)+\alpha_{t 1} \sum_{j=1}^{n} \sum_{l=0}^{\sigma_{q j}} \sum_{s=k-l}^{k-1} b_{q j l}(k+l) x_{j}(s) \\
& \\
& \quad+\alpha_{t 1} \sum_{s=k-\tau_{q}}^{k-1} d_{q}\left(k+\tau_{q}\right) u_{q}(s)-\alpha_{t 2} \sum_{j=1}^{n} \sum_{s=k-\tau_{t j}}^{k-1} a_{t j}\left(k+\tau_{t j}\right) x_{j}(s) \\
& \\
& \quad-\alpha_{t 2} \sum_{j=1}^{n} \sum_{l=0}^{\sigma_{t j}} \sum_{s=k-l}^{k-1} b_{t j l}(k+l) x_{j}(s)-\alpha_{t 2} \sum_{s=k-\tau_{t}}^{k-1} d_{t}\left(k+\tau_{t}\right) u_{t}(s) \\
&\left.+\alpha_{t 3} \sum_{s=k-\delta_{q}}^{k-1} g_{q}\left(k+\delta_{q}\right) x_{q}(s)-\alpha_{t 4} \sum_{s=k-\delta_{t}}^{k-1} g_{t}\left(k+\delta_{t}\right) x_{t}(s)\right\}
\end{aligned}
$$


Calculating $V_{t}(k+1) / V_{t}(k)$, similarly to $V_{n}(k)$, we can obtain

$$
\begin{aligned}
& \frac{V_{t}(k+1)}{V_{t}(k)}=\exp \left\{-\alpha_{t 1} r_{q}(k)+\alpha_{t 2} r_{t}(k)\right. \\
& \text { - }\left(\alpha_{t 3} e_{q}(k)-\alpha_{t 1} D_{q}(k)\right) u_{q}(k) \\
& +\left(-\alpha_{t 2} D_{t}(k)+\alpha_{t 4} e_{t}(k)\right) u_{t}(k) \\
& +\sum_{j \neq q, t}^{n-1}\left(\alpha_{t 1} A_{q j}(k)-\alpha_{t 2} A_{t j}(k)\right) x_{j}(k) \\
& +\left(\alpha_{t 1} A_{q q}(k)-\alpha_{t 2} A_{t p}(k)-\alpha_{t 2} R_{q}(k)\right) x_{q}(k) \\
& \left.+\left(\alpha_{t 1} A_{q t}(k)-\alpha_{t 2} A_{t t}(k)-\alpha_{t 4} R_{t}(k)\right) x_{t}(k)\right\}
\end{aligned}
$$

for all $k \geq K_{t}$. From inequalities (3.26)-(3.30), we further obtain

$$
V_{t}(k+1) \leq V_{t}(k) \exp \left\{-\alpha_{t 1} r_{q}(k)+\alpha_{t 2} r_{t}(k)+P(k)\right\}
$$

for all $k \geq K_{t}$, where

$$
P(k)=\sum_{j=t+1}^{n}\left(\alpha_{t 1} A_{q j}(k)+\alpha_{t 2} A_{t j}(k)\right) x_{j}(k)
$$

From (3.19) we have $P(k) \rightarrow 0$ as $k \rightarrow \infty$. Hence,

$$
\lim _{k \rightarrow \infty} \sum_{s=k}^{k+\omega-1} P(s)=0
$$

Thus, from (3.22) we can obtain that there exists an integer $K_{t}^{*} \geq K_{t}$ such that

$$
\sum_{s=k}^{k+\omega-1}\left(-\alpha_{\mathrm{t} 1} r_{q}(s)+\alpha_{t 2} r_{t}(s)+P(s)\right)<-\frac{1}{2} \varepsilon_{t} \alpha_{n 2} \eta_{0}
$$

for all $k>K_{t}^{*}$. By calculating, from (3.33) we obtain

$$
V_{t}(k) \leq V_{t}\left(K_{t}^{*}\right) \exp \left\{\sum_{s=K_{t}^{*}}^{k-1}\left(-\alpha_{t 1} r_{q}(s)+\alpha_{t 2} r_{t}(s)+P(s)\right)\right\}
$$


From this, a similar argument as in the proof of (3.15)-(3.19), we further can obtain

$$
x_{t}(k) \leq Q_{t}^{*} \exp \left\{-\lambda_{t}^{*} k\right\} \quad \forall k \geq K_{t}^{*}
$$

where $Q_{t}^{*}$ and $\lambda_{t}^{*}$ are two positive constants. From (3.38), we finally obtain

$$
\lim _{k \rightarrow \infty} x_{t}(k)=0, \quad \sum_{k=0}^{\infty} x_{t}(k)<\infty
$$

Next, we consider the second equation of system (1.8), applying Lemma 2.3 we can easily obtain $u_{t}(k) \rightarrow 0$ as $k \rightarrow \infty$.

Finally, according to the induction principle, we have

$$
\lim _{k \rightarrow \infty} x_{i}(k)=0, \quad \lim _{k \rightarrow \infty} u_{i}(k)=0, \quad \sum_{k=0}^{\infty} x_{i}(k)<\infty \quad \forall i>r .
$$

This completes the proof of Theorem 3.1.

As consequences of Theorem 3.1, we consider the following two special cases of system (1.8).

Case 1. Nondelayed nonautonomous discrete $n$-species Lotka-Volterra competitive systems with feedback controls

$$
\begin{aligned}
& x_{i}(k+1)=x_{i}(k) \exp \left\{r_{i}(k)-\sum_{j=1}^{n} a_{i j}(k) x_{j}(k)-d_{i}(k) u_{i}(k)\right\}, \\
& u_{i}(k+1)=\left(1-e_{i}(k)\right) u_{i}(k)+g_{i}(k) x_{i}(k), \quad i=1,2, \ldots, n .
\end{aligned}
$$

For system (3.41), assumptions $\left(H_{1}\right)$ and $\left(H_{3}\right)$ become into the following form

$\left(H_{1}^{\prime}\right) r_{i}(k)$ is a bounded sequence defined on $Z ; a_{i j}(k), d_{i}(k)$ and $g_{i}(k)(i, j=1,2, \ldots, n)$ are nonnegative bounded sequences defined on $Z$.

$\left(H_{3}^{\prime}\right)$ There exist positive integers $\omega$ and $\lambda$ such that for each $i=1,2, \ldots, n$

$$
\liminf _{n \rightarrow \infty} \sum_{s=n}^{n+\omega-1} r_{i}(s)>0, \quad \liminf _{n \rightarrow \infty} \sum_{s=n}^{n+\lambda-1} a_{i i}(s)>0
$$

Directly from Theorem 3.1, we have the following corollary. 
Corollary 3.2. Assume that assumptions $\left(H_{1}^{\prime}\right),\left(H_{2}\right),\left(H_{3}^{\prime}\right)$ and $\left(H_{4}\right)$ hold and there exists a integer $1 \leq r<n$ such that for any $h>r$ there exists an integer $i_{h}<h$ such that

$$
\begin{gathered}
\limsup _{k \rightarrow \infty} \frac{\sum_{s=k}^{k+\omega-1} r_{h}(s)}{\sum_{s=k}^{k+\omega-1} r_{i_{h}}(s)}<\liminf _{k \rightarrow \infty} \frac{a_{h j}(k)}{a_{i_{h} j}(k)} \quad \forall j \leq h, \\
\liminf _{k \rightarrow \infty} \frac{d_{h}(k)}{e_{h}(k)}>\limsup _{k \rightarrow \infty}\left(\frac{a_{i_{h} h}(k)}{g_{h}(k)} \limsup _{k \rightarrow \infty} \frac{\sum_{s=k}^{k+\omega-1} r_{h}(s)}{\sum_{s=k}^{k+\omega-1} r_{i_{h}}(s)}-\frac{a_{h h}(k)}{g_{h}(k)}\right), \\
\limsup _{k \rightarrow \infty} \frac{d_{i_{h}}(k)}{e_{i_{h}}(k)}<\liminf _{k \rightarrow \infty}\left(\frac{a_{h_{h}}(k)}{g_{i_{h}}(k)} \liminf _{k \rightarrow \infty} \frac{\sum_{s=k}^{k+\omega-1} r_{i_{h}}(s)}{\sum_{s=k}^{k+\omega-1} r_{h}(s)}-\frac{a_{i_{h} i_{h}}(k)}{g_{i_{h}}(k)}\right),
\end{gathered}
$$

then for each $i=r+1, \ldots, n$ one has

$$
\lim _{k \rightarrow \infty} x_{i}(k)=0, \quad \lim _{k \rightarrow \infty} u_{i}(k)=0, \quad \sum_{k=0}^{\infty} x_{i}(k)<\infty
$$

for any positive solution $\left(x_{1}(k), x_{2}(k), \ldots, x_{n}(k), u_{1}(k), u_{2}(k), \ldots, u_{n}(k)\right)$ of system (3.41).

Case 2. Pure delayed nonautonomous discrete $n$-species Lotka-Volterra competitive systems without feedback controls

$$
x_{i}(k+1)=x_{i}(k) \exp \left\{r_{i}(k)-\sum_{j=1}^{n} a_{i j}(k) x_{j}\left(k-\tau_{i j}\right)-\sum_{j=1}^{n} \sum_{l=0}^{\sigma_{i j}} b_{i j l}(k) x_{j}(k-l)\right\}, \quad i=1,2, \ldots, n .
$$

For system (3.41), assumption $\left(H_{1}\right)$ becomes into the following form.

$\left(H_{1}^{\prime \prime}\right) r i(k)$ is a bounded sequence defined on $Z ; a_{i j}(k)$ and $b_{i j l}(k)(i, j=1,2, \ldots, n, l=$ $\left.0,1, \ldots, \sigma_{i j}\right)$ are nonnegative bounded sequences defined on $Z ; \tau_{i j}$ and $\sigma_{i j}(i, j=$ $1,2, \ldots, n)$ are nonnegative integers.

Directly from Theorem 3.1, we have the following corollary.

Corollary 3.3. Assume that assumptions $\left(H_{1}^{\prime \prime}\right)$ and $\left(H_{3}\right)$ hold and there exists an integer $1 \leq r<n$ such that for any $h>r$ there exists an integer $i_{h}<h$ such that

$$
\limsup _{k \rightarrow \infty} \frac{\sum_{s=k}^{k+\omega-1} r_{h}(s)}{\sum_{s=k}^{k+\omega-1} r_{i_{h}}(s)}<\liminf _{k \rightarrow \infty} \frac{A_{h j}(k)}{A_{i_{h} j}(k)} \quad \forall j \leq h,
$$

then for each $i=r+1, \ldots, n$ one has

$$
\lim _{k \rightarrow \infty} x_{i}(k)=0, \quad \sum_{k=0}^{\infty} x_{i}(k)<\infty
$$

for any positive solution $\left(x_{1}(k), x_{2}(k), \ldots, x_{n}(k)\right)$ of system (3.45). 
Remark 3.4. By comparison, we easily see that the results obtained in this paper are a very good extension of the corresponding results obtained in [9] on the extinction of species for nonautonomous continuous Lotka-Volterra competitive system with pure-delays and feedback controls to discrete ones.

\section{An Example}

We consider the following periodic discrete three-species Lotka-Volterra competitive system with pure-delays and feedback controls:

$$
\begin{gathered}
x_{i}(k+1)=x_{i}(k) \exp \left\{r_{i}(k)-\sum_{j=1}^{3} a_{i j}(k) x_{j}\left(k-\tau_{i j}\right)-d_{i}(k) u_{i}\left(k-\tau_{i}\right)\right\}, \\
u_{i}(k+1)=\left(1-e_{i}(k)\right) u_{i}(k)+g_{i}(k) x_{i}\left(k-\delta_{i}\right), \quad i=1,2,3 .
\end{gathered}
$$

where

$$
\begin{array}{ll}
r_{1}(k)=\frac{1}{2}+\sin \left(\frac{1}{3}+\frac{\pi}{2} k\right), & r_{2}(k)=\frac{1}{2}+\sin \left(\frac{1}{4}+\frac{\pi}{2} k\right), \\
r_{3}(k)=3\left(\frac{1}{4}+\sin \left(\frac{1}{5}+\frac{\pi}{2} k\right)\right), & a_{11}(k)=\frac{1}{3}\left(1+\cos ^{2}\left(\frac{1}{3}+\frac{\pi}{2} k\right)\right), \\
a_{12}(k)=\frac{1}{6}+\sin ^{2}\left(\frac{1}{3}+\frac{\pi}{2} k\right), & a_{13}(k)=\frac{1}{4}+\cos ^{2}\left(\frac{1}{3}+\frac{\pi}{2} k\right), \\
a_{21}(k)=1+\cos ^{2}\left(\frac{1}{3}+\frac{\pi}{2} k\right), & a_{22}(k)=\frac{1}{2}+\cos ^{2}\left(\frac{1}{3}+\frac{\pi}{2} k\right), \\
a_{31}(k)=4+\sin ^{2}\left(\frac{1}{3}+\frac{\pi}{2} k\right), & a_{32}(k)=\frac{9}{2}+\sin ^{2}\left(\frac{1}{3}+\frac{\pi}{2} k\right), \\
a_{33}(k)=8+\sin ^{2}\left(\frac{1}{3}+\frac{\pi}{2} k\right), & d_{1}(k)=\frac{1}{10}\left(1+\sin ^{2}\left(\frac{1}{3}+\frac{\pi}{2} k\right)\right), \\
d_{2}(k)=\frac{1}{2}+\sin ^{2}\left(\frac{1}{3}+\frac{\pi}{2} k\right), & d_{3}(k)=2+\cos ^{2}\left(\frac{1}{3}+\frac{\pi}{2} k\right), \\
e_{1}(k)=\frac{1}{3}\left(1+\cos ^{2}\left(\frac{1}{3}+\frac{\pi}{2} k\right)\right), & e_{2}(k)=\frac{1}{4}\left(1+\sin ^{2}\left(\frac{1}{3}+\frac{\pi}{2} k\right)\right), \\
e_{3}(k)=\frac{1}{3}\left(1+\sin ^{2}\left(\frac{1}{3}+\frac{\pi}{2} k\right)\right), & g_{1}(k)=\frac{1}{3}\left(1+\cos ^{2}\left(\frac{1}{3}+\frac{\pi}{2} k\right)\right), \\
g_{2}(k)=1+\cos ^{2}\left(\frac{1}{3}+\frac{\pi}{2} k\right), & g_{3}(k)=1+\cos ^{2}\left(\frac{1}{3}+\frac{\pi}{2} k\right) .
\end{array}
$$


Clearly, in system (4.2), $r=2, h=3$, and $i_{h}=1$. By calculating, we obtain

$$
\begin{aligned}
& \limsup _{k \rightarrow \infty} \frac{\sum_{s=k}^{k+3} r_{3}(s)}{\sum_{s=k}^{k+3} r_{1}(s)}=\limsup _{k \rightarrow \infty} \frac{\sum_{s=k}^{k+3} 3(1 / 4+\sin (1 / 5+(\pi / 2) s))}{\sum_{s=k}^{k+3}[1 / 2+\sin (1 / 3+(\pi / 2) s)]}=\frac{3}{2}, \\
& \liminf _{k \rightarrow \infty} \frac{\sum_{s=k}^{k+3} r_{1}(s)}{\sum_{s=k}^{k+3} r_{3}(s)}=\liminf _{k \rightarrow \infty} \frac{\sum_{s=k}^{k+3}[1 / 2+\sin (1 / 3+(\pi / 2) s)]}{\sum_{s=k}^{k+3} 3(1 / 4+\sin (1 / 5+(\pi / 2) s))}=\frac{2}{3}, \\
& \liminf _{k \rightarrow \infty} \frac{A_{33}(k)}{A_{13}(k)}=\liminf _{k \rightarrow \infty} \frac{8+\sin ^{2}\left(1 / 3+(\pi / 2)\left(k+\tau_{33}\right)\right)}{1 / 4+\cos ^{2}\left(1 / 3+(\pi / 2)\left(k+\tau_{13}\right)\right)} \geq \frac{32}{5}, \\
& \liminf _{k \rightarrow \infty} \frac{A_{32}(k)}{A_{12}(k)}=\liminf _{k \rightarrow \infty} \frac{9 / 2+\sin ^{2}\left(1 / 3+(\pi / 2)\left(k+\tau_{32}\right)\right)}{1 / 6+\sin ^{2}\left(1 / 3+(\pi / 2)\left(k+\tau_{13}\right)\right)} \geq \frac{54}{14}, \\
& \liminf _{k \rightarrow \infty} \frac{A_{31}(k)}{A_{11}(k)}=\liminf _{k \rightarrow \infty} \frac{4+\sin ^{2}\left(1 / 3+(\pi / 2)\left(k+\tau_{31}\right)\right)}{1 / 3\left(1+\cos ^{2}\left(1 / 3+(\pi / 2)\left(k+\tau_{11}\right)\right)\right)} \geq 6, \\
& \limsup _{k \rightarrow \infty}\left(\frac{A_{13}(k)}{R_{3}(k)} \limsup _{k \rightarrow \infty} \frac{\sum_{s=k}^{k+3} r_{3}(s)}{\sum_{s=k}^{k+3} r_{1}(s)}-\frac{A_{33}(k)}{R_{3}(k)}\right) \\
& =\limsup _{k \rightarrow \infty} \frac{3 / 2\left(1 / 4+\cos ^{2}\left(1 / 3+(\pi / 2)\left(k+\tau_{13}\right)\right)\right)-\left(8+\sin ^{2}\left(1 / 3+(\pi / 2)\left(k+\tau_{33}\right)\right)\right)}{1+\cos ^{2}\left(1 / 3+(\pi / 2)\left(k+\delta_{3}\right)\right)} \\
& \leq \frac{3 / 2(1 / 4+1)-8}{2}<0, \\
& \liminf _{k \rightarrow \infty}\left(\frac{A_{31}(k)}{R_{1}(k)} \liminf _{k \rightarrow \infty} \frac{\sum_{s=k}^{k+3} r_{1}(s)}{\sum_{s=k}^{k+3} r_{3}(s)}-\frac{A_{11}(k)}{R_{1}(k)}\right) \\
& =\liminf _{k \rightarrow \infty} \frac{2 / 3\left(4+\sin ^{2}\left(1 / 3+(\pi / 2)\left(k+\tau_{31}\right)\right)\right)-\left(1 / 3\left(1+\cos ^{2}\left(1 / 3+(\pi / 2)\left(k+\tau_{11}\right)\right)\right)\right)}{1 / 3\left(1+\cos ^{2}\left(1 / 3+(\pi / 2)\left(k+\delta_{1}\right)\right)\right)} \\
& \geq \frac{(2 / 3) 4-(1 / 3) 2}{2 / 3}=3 \text {, } \\
& \liminf _{k \rightarrow \infty} \frac{D_{3}(k)}{e_{3}(k)}=\liminf _{k \rightarrow \infty} \frac{2+\cos ^{2}\left(1 / 3+(\pi / 2)\left(k+\tau_{3}\right)\right)}{1 / 3\left(1+\sin ^{2}(1 / 3+(\pi / 2) k)\right)} \geq 3, \\
& \limsup _{k \rightarrow \infty} \frac{D_{1}(k)}{e_{1}(k)}=\limsup _{k \rightarrow \infty} \frac{1 / 10\left(1+\sin ^{2}\left(1 / 3+(\pi / 2)\left(k+\tau_{1}\right)\right)\right)}{1 / 3\left(1+\cos ^{2}(1 / 3+(\pi / 2) k)\right)} \leq \frac{3}{5} .
\end{aligned}
$$

From these inequalities we see that all conditions (3.2) in Theorem 3.1 hold. In addition, we also see that assumptions $\left(H_{1}\right)-\left(H_{4}\right)$ obviously hold. Therefore, by Theorem 3.1 we obtain that species $x_{3}$ in system (4.3) is extinct. 


\section{Acknowledgment}

This work was supported by the National Natural Science Foundation of China (10961022, 10901130), the Scientific Research Programmes of Colleges in Xinjiang (XJEDU2007G01, XJEDU2006I05, XJEDU2008S10), and the National Key Technologies R \& D Program of China (2008BAI68B01) and the Foundation of Education Committee of Jiangxi (GJJ0931).

\section{References}

[1] R. May, "Biological population with nonoverlapping generations: stable points, stable cycles and chaos," Science, vol. 186, pp. 645-647, 1974.

[2] Z. Lu and W. Wang, "Permanence and global attractivity for Lotka-Volterra difference systems," Journal of Mathematical Biology, vol. 39, no. 3, pp. 269-282, 1999.

[3] Y. Chen and Z. Zhou, "Stable periodic solution of a discrete periodic Lotka-Volterra competition system," Journal of Mathematical Analysis and Applications, vol. 277, no. 1, pp. 358-366, 2003.

[4] Q. Zhang and Z. Zhou, "Permanence of a nonautonomous Lotka-Volterra difference system with delays," Mathematical Sciences Research Journal, vol. 7, no. 3, pp. 99-106, 2003.

[5] W. Wang, G. Mulone, F. Salemi, and V. Salone, "Global stability of discrete population models with time delays and fluctuating environment," Journal of Mathematical Analysis and Applications, vol. 264, no. 1, pp. 147-167, 2001.

[6] Y. Muroya, "Persistence and global stability for discrete models of nonautonomous Lotka-Volterra type," Journal of Mathematical Analysis and Applications, vol. 273, no. 2, pp. 492-511, 2002.

[7] Y. Muroya, "Persistence and global stability in discrete models of Lotka-Volterra type," Journal of Mathematical Analysis and Applications, vol. 330, no. 1, pp. 24-33, 2007.

[8] X. Liao, S. Zhou, and Y. Chen, "Permanence and global stability in a discrete $n$-species competition system with feedback controls," Nonlinear Analysis: Real World Applications, vol. 9, no. 4, pp. 1661$1671,2008$.

[9] H. Hu, Z. Teng, and S. Gao, "Extinction in nonautonomous Lotka-Volterra competitive system with pure-delays and feedback controls," Nonlinear Analysis: Real World Applications, vol. 10, no. 4, pp. 2508-2520, 2009.

[10] H. Hu, Z. Teng, and H. Jiang, "On the permanence in non-autonomous Lotka-Volterra competitive system with pure-delays and feedback controls," Nonlinear Analysis: Real World Applications, vol. 10, no. 3, pp. 1803-1815, 2009.

[11] J. Xu and Z. Teng, "Almost sufficient and necessary conditions for permanece and extinction of nonautonomous discrete logistic systems with time-varying delays and feedback," Submitted to Applications of Mathematics. 


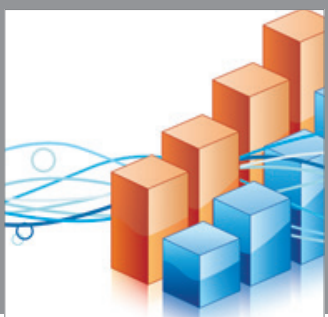

Advances in

Operations Research

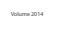

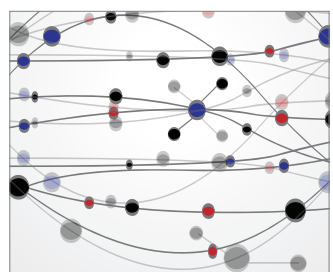

\section{The Scientific} World Journal
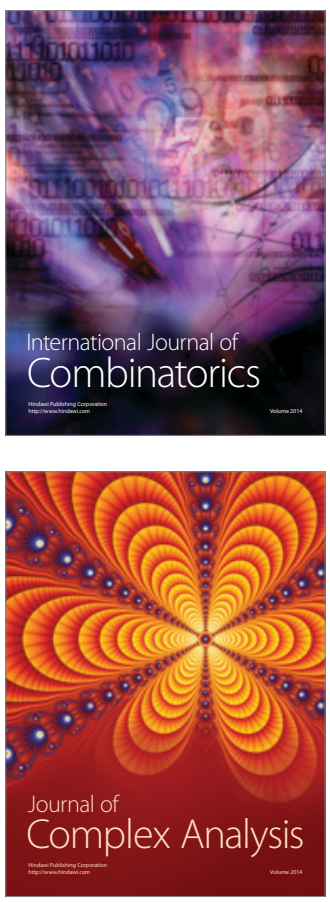

International Journal of

Mathematics and

Mathematical

Sciences
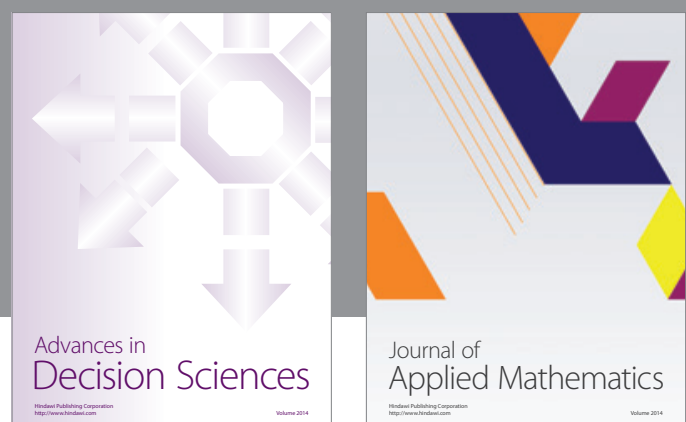

Journal of

Applied Mathematics
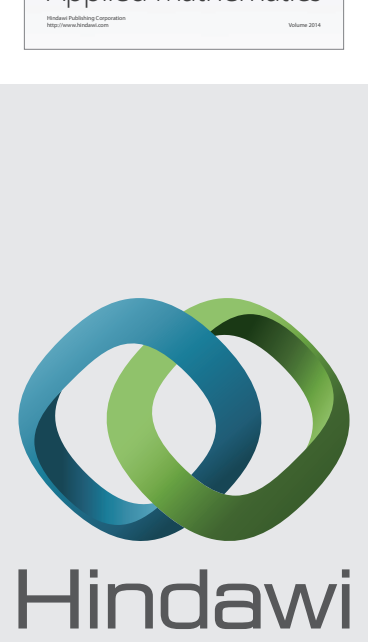

Submit your manuscripts at http://www.hindawi.com
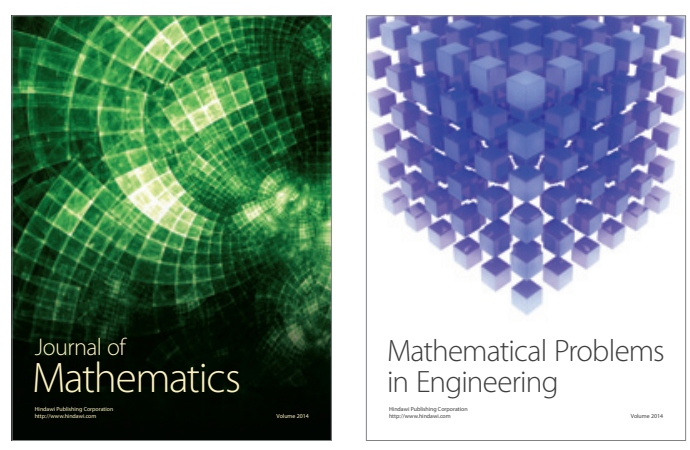

Mathematical Problems in Engineering
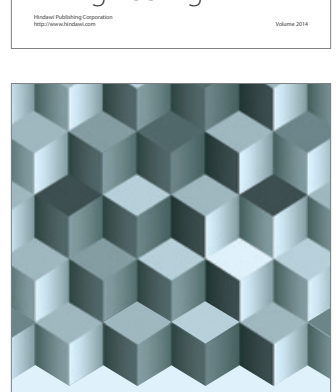

Journal of

Function Spaces
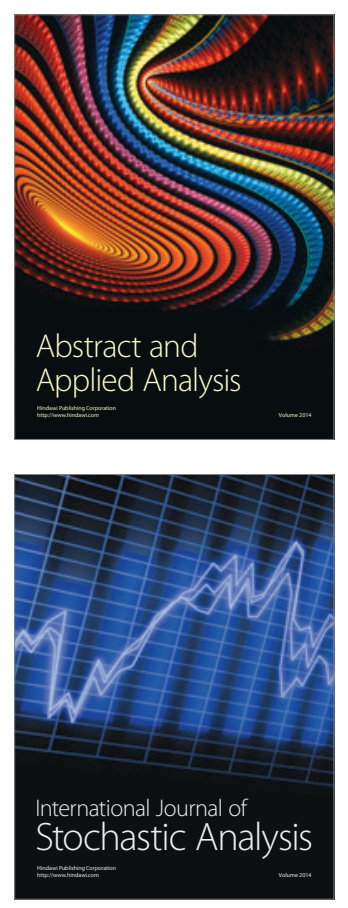

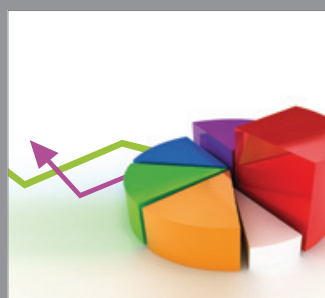

ournal of

Probability and Statistics

Promensencen
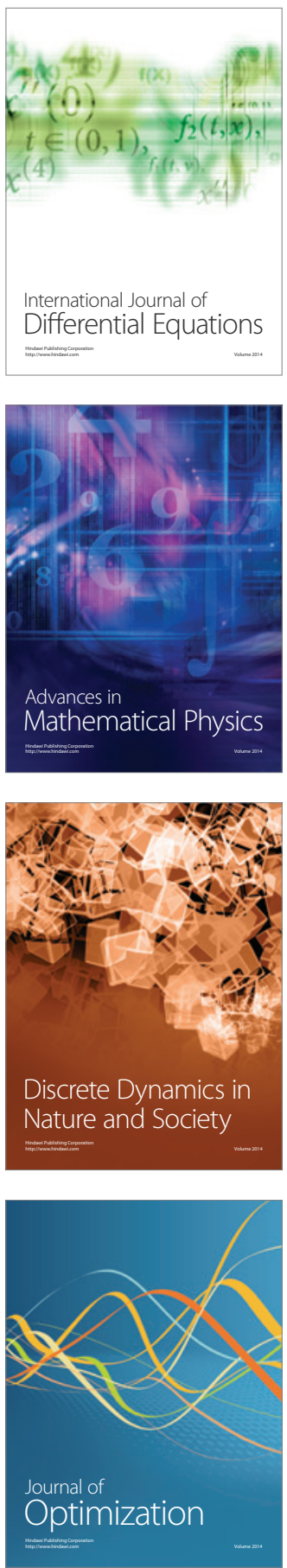